\title{
Anatomical and Clinical Implication of Corona Mortis in the Anterior Approaches of Pelvis in Sudanese Patient
}

\author{
Azer Abbas ${ }^{1}$, Mohamed Hamid Awadelseid ${ }^{2}$, Mohamed Alfath Abdalgawi ${ }^{3}$ \\ ${ }^{1}$ Departement of Orthopedic Excellence Trauma Centre-Khartoum, Khartoum, Sudan \\ ${ }^{2}$ Departement of Orthopedic Alazhary University-Khartoum, Khartoum, Sudan \\ ${ }^{3}$ Department of Orthopedic Military Hospital-Khartoum, Khartoum, Sudan \\ Email: mhawad3055@gmail.com
}

How to cite this paper: Abbas, A., Awadelseid, M.H. and Abdalgawi, M.A. (2021) Anatomical and Clinical Implication of Corona Mortis in the Anterior Approaches of Pelvis in Sudanese Patient. Open Journal of Orthopedics, 11, 85-95.

https://doi.org/10.4236/ojo.2021.113009

Received: December 13, 2020

Accepted: March 14, 2021

Published: March 17, 2021

Copyright (c) 2021 by author(s) and Scientific Research Publishing Inc. This work is licensed under the Creative Commons Attribution International License (CC BY 4.0).

http://creativecommons.org/licenses/by/4.0/

\begin{abstract}
Background: Corona Mortis is an abnormal blood vessels connecting Between obturator and external iliac systems of vessels and inferior epigastric vessels. It is located behind the superior public ramus at a variable distance from the symphysis pubis. Orthopaedic surgeons and obestitrtion found these vessels in the field of surgery. So displaced fractures or iatrogenic injury, can cause life threatening bleeding and hence these vascular anomalies are referred to us crown of death. The bulk of the studies about the corona mortise were conducted in the cadavers or 3D CT angiography and there was no studies were conducted about corona mortise in Sudan. The purpose of this study was to study the corona mortis anatomical and clinical implication in the anterior approaches to the pelvis and acetabulum in Sudanese patient. Methodology: Hospital based descriptive cross-sectional study was conducted among patients sustaining anterior pelvic anterior acetabulum fracture and underwent open reduction internal fixation by reconstruction plates. This study was conducted in Bahri Teaching Hospital from June 2017 to June 2018. Through the most common anterior approaches to the pelvis and acetabulum (Stoppa-modified stoppa-ilioinguinal), done by single surgeon and fulfil data sheet intra-operatively. Results: In a total of 30 pelvic halves corona mortis was detected in 13 patients (43\%). The Majority in the Rt.hemipelvies 7 (53.8\%) specimens: arterial 4/13 (30.8\%), venous $1 / 13$ (7.7\%) and both arterial and venous in $8 / 13(61.5 \%)$ specimens respectively. Of these $(53.9 \%)$ had a large Diameter $>5 \mathrm{~mm}$ and the largest one measure $80 \mathrm{~mm}$, with mean distance From the symphysis $5.8+1.6 \mathrm{~mm}$. Conclusion: Only 13 patients have corona mortis, 12 of them were arterial with diameter more than $5 \mathrm{~mm}$ (53\%), and thus surgeon should exercise caution during Surgery and secure
\end{abstract}


its site to help minimizing surgery time, Clear surgical field and prevent iatrgenic injury.

\section{Keywords}

Corona Mortis, Anterior Approaches, Pelvis

\section{Introduction}

In cadaveric study done by S. Darmanis [1], to identify whether the corona mortise actually (in clinical practice) is a threat in the anterior approach to the pelvis and acetabulum. Or if more ink than blood has been spilt over this anatomical variant, which was carried in forty cadavers (27 male and 13 female) (80 cadavers hemipelvies).

Bilateral illioinguinal approach were carried out in 80 hemipelvies. A vascular communication (>1 - $2 \mathrm{~mm}$ in diameter) was found in 66 hemipelvies (83\%). In 2 speciments (2.5\%) a large diameter vessels was present (4 or more millimeter). in one case the large diameter vessels was a corona mortise artery running almost vertically behind the superior public ramus connecting the external iliac artery with the obturator artery at $5.2 \mathrm{~cm}$ lateral to the symphysis pubis. in the other case the large vessels was an aberrant obturator artery, which originated from the external iliac artery behind the superior public ramus entering the obturator foramen vertically.

\section{Arterial corona mortise}

Of the 80 cadaveric hemipelvies, 29 (36\%) had an arterial connection. multiple arterial anastomoses were found in only one speciments. the average distance of the connecting artery from the symphysis pubis was $71 \mathrm{~mm}(42-88 \mathrm{~mm})$. in all the hemipelvies the artery passed vertically to the upper surface of the superior pubic ramus forming a "vascular arch" around the superior pubic ramus. a few variation were observed where the arterial connection originated more posteriorly and passed antero-inferiorly on the lateral wall of the pelvis and connected with the obturator artery as it entered the obturator foramen.

\section{Venous corona mortis:}

Venous anastomoses were more common than arterial. avenous connection was found in 48 speciments (60\% hemipelvies). The avenge distance of the connecting vein from the symphysis pubic was $65 \mathrm{~mm}(39-82 \mathrm{~mm})$. The anastomoses vein passed over the superior pubic ramus in close proximity to the femoral ring and the lacunar ligament. It passed medial to the iliopectineal eminence and the iliopsoas muscle. It then descended vertically next to the artery, if present and finally connected to the obturator vein.

\section{Aberrant obturator vessels:}

In one hemipelvies an aberrant obturator artery originating from the external iliac artery was identified. In three hemipelvies there was an aberrant obturator vein originating from the external iliac vein. In the four hemipelvies, these aber- 
rant obturator vessels all entered the obturator foramen at the same point as the classical corona mortis. Their origin was always more posterior at an acute angle crossing the superior pubic ramus. In one case the aberrant obturator artery passed lateral onto the femoral ring and the pectineal ligament and the descended into the pelvis. The mean distance of the aberrant obturator vessels crossing the superior edge of the superior pubic ramus was $12 \mathrm{~mm}$ (range of 9 $14 \mathrm{~mm}$ ) posterior to the average distance of corona mortis from the symphysis pubic. So they concluded that a vascular connection between the obturator and the external iliac or inferior epigastric system is located over the superior pubic ramus in high percentage of cadaveric dissection. Its diameter as well as it course varies, and accidental transaction result in a potentially life-threatening hemorrhage. Never the less, in clinical practice the incidence of an excessive hemorrhage from these variant vessels is extremely low. Surgeons who carry out an anterior approach to the acetabulum have to be cautious about encountering a leash of vessels at around $6 \mathrm{~cm}$ (range $40-96 \mathrm{~mm}$ ) lateral to the symphysis pubis. This possible danger does not seem to be a significant clinical risk and should not compromise the surgical decision to operate through the illioinguinal approach for fear of excessive hemorrhage [1].

Hong HX, et al. [2], in their study about corona mortis and its clinical significance done in 50 cadavers hemipelvies found that $72 \%$ of the cadavers sides had at least one communicating vessels between the obturator system and the external iliac or inferior epigastric system on the superior pubic ramus. The average diameter of the connecting vessels was $2.6 \mathrm{~mm}$ (range $2.0-4.2 \mathrm{~mm}$ ). It coursed over the superior pubic ramus or iliopubic eminence vertically to enter the obturator foramen and exit the pelvis. The average distance from pubic symphysis to the vascular connection between the obturator and external iliac system was $52 \mathrm{~mm}$ (range $38-68 \mathrm{~mm}$ ).

Their conclusion is that a vascular connection between the obturator system and the external iliac or inferior epigastric system were found over the superior pubic ramus with a high incidence. They are prone to damaged during the ilioinguinal approach as an anterior approach to the acetabulum and pelvis. Thus, corona mortis located over the superior pubic ramus deserves great attention during the ilioinguinal approach.

OKcu G, et al. [3] conducted a cadaveric study about corona mortis incidence and location in 75 cadaveric. they found a vascular communication in 91 (61\%) cadaver side. venous anastomoses were much commoner. 78 side $(52 \%)$ had venous connection. arterial connection were found in 29 cases (19\%). 21 of 75 pelvises had bilateral vascular connection, but only 9 had bilateral mirror image vascular trees. a vascular connection was seen in 70 sides of 58 male specimens and in 21 sides of 17 female specimens. venous connection were found in 36 male specimens, a rate of 0.6 ( $95 \%$ CI $0.5 \%-0.7 \%)$ and in 11 female specimens, rate 0.7 ( $95 \%$ CI $0.4 \%-0.8 \%, \mathrm{p}=0.8$ ).

Arterial anastomoses were seen in 16 male specimens, rate 0.3 (95\% CI $0.2 \%$ $0.4 \%)$ and in 3 female specimens rate 0.2 ( $95 \%$ CI $0.05 \%-0.4 \%, p=0.4)$. The 
average distance of the arterial anastomoses from the symphysis pubic was 64 (45 - 90) $\mathrm{mm}$ and $56(37-80) \mathrm{mm}$ from the communicating veins. They found no statistically significant difference between the genders [4].

Another study about anatomical consideration on corona mortise done by Mugurel CR, et al. in 20 human adult cadavers (12 male and 8 female); bilateral dissection of the 40 hemipelvies (termed here specimens) were performed using the anterior-superior approach. they found the corona mortis was identified on the superior pubic branch in $32(\mathrm{n})$ of the $40(\mathrm{n})$ hemipelvies dissected (80\%). in what concerns the vascular composition of corona mortis, their results mainly suggested there is a wide variability of it.

They identified 26 hemipelvies ( $65 \%$ of 40 pelvic halves, $81.25 \%$ of the 32 specimens positive for the presence of the corona mortis) with arterial elements crossing over the superior pubic branch and 22 hemipelvies (55\% of the 40 pelvic halves, $68.75 \%$ of the 32 specimens positive for the presence of the corona mortis) with venous elements at the levels.

\section{General objectives:}

To study the Corona mortis anatomical and clinical implication in the anterior approaches to the pelvis and acetabulum.

\section{Specific objectives:}

1) To identify the aberrant vessel location and vascularity.

2) To determine the risk of intra complications (massive bleeding).

3) To rate frequency of complication.

4) To assess control procedures.

\section{Material and Methods}

Study design: Descriptive cross-section hospital based study was conducted in Bahri Teaching Hospital that is located in Khartoum $1^{\text {ST }}$ Of June $2017-30^{\text {th }}$ of June 2018 All 30 patients sustaining anterior pelvic and anterior acetabulum fracture and underwent open Reduction Internal fixation by reconstruction plates who were admitted to Bahri Teaching Hospital during the study period. Standard predesigned structurd questionnaire was filled in direct interview with patient, intraoperative finding chick list was completed by author. Data mange and analysis by SPSS version 21 .

Patients with these criteria were excluded:

1) Previous similar surgery.

2) Patients with previous obstetric gynaecological procedure (pelvic hysterectomy).

3) Open pelvic injuries.

\section{Stoppa Approach to Acetabulum:}

Indications in acetabular fractures, pelvic ring injuries Approach provides access to pubic body, superior pubic ramus pubic root, ilium above and below the pectineal line, quadrilateral plate medial aspect of the posterior column. Sciatic buttress. Anterior sacroiliac joint, upper ilium and iliac crest. 


\section{Preparation \& Positioning}

a radiolucent table is required, Foley catheter is required to improve visualization, Position supine ipsilateral limb is draped free into the field, hip and knee are flexed to relax the ilipsoas/femoral neurovascular bundle. Operating surgeon is on the opposite side of the table

\section{Approach}

A transverse incision is made approximately $2 \mathrm{~cm}$ above the symphysis this is carried short of each external inguinal ring for the "lateral window", an incision is made along the iliac crest, starting $\sim 2 \mathrm{~cm}$ posterior to the ASIS, following the iliac crest posteriorly subcutaneous tissue and rectus fascia are incised transversely the pyrimidalis muscle is released and tagged for later repair, the rectus abdominus fascia is split along the linea alba. The transversalis fascia is opened superior to the pubic symphysis this opens the potential space of Retzius (space behind the symphysis and anterior to the bladder) for the "lateral window", the insertion of the external oblique is released, permitting dissection into the internal iliac crest fossa (requires elevation of the iliacus muscle.

The insertion of the rectus abdominus muscle is released off the posterior pubic rami but maintained anteriorly, a Hohmann retractor is used to retract the rectus anteriorly, the iliopectineal fascia is released to enter the true pelvis, anastamoses between the external iliac and obturator vessels (corona mortis) should be identified along the superior pubic ramus and ligated, the iliopsoas can now be subperiosteally elevated, and a retractor is used to retract the iliopsoas and external iliac vessels, the entire pelvic brim should be visualized at this time.the obturator neurovascular bundle is exposed and protected as the quadrilateral surface and posterior column are dissected [5].

\section{Ilioinguinal Approach}

- Position supine with greater troch on side of fracture at edge of table place bump under ipsilateral buttock, flex affected leg to relax iliopsoas and neurovascular structures.

- Imaging ensure clear fluoroscopic images can be obtained prior to draping

- Insert catheter to empty bladder (will obscure vision).

- Incision begins at midline $3-4 \mathrm{~cm}$ proximal to symphysis pubis.proceeds laterally to ASIS, then along anterior 2/3's of iliac crest, extend incision beyond most convex portion of ilium.

- Superficial Dissection dissect through subcutaneous fat, start laterally, incise periosteum along iliac crest, release abdominal and iliacus muscle insertions from ilium.superiosteally elevate iliacus from internal iliac fossa to SI joint and pelvic brim, pack internal iliac fossa for hemostasis, through lower portion of incision expose aponeurosis of external oblique and rectus abdominus, divide exposed aponeurosis in line with skin incision one $\mathrm{cm}$ proximal to external inguinal ring, will often have to sacrifice lateral cutaneous nerve of the thigh, thus unroofs inguinal canal, and exposes inguinal ligament, identify and protect ilioinguinal nerve, isolate spermatic cord/round ligament and place penrose around structures to retract, sharply incise inguinal liga- 
ment, leaving 1 - 2 mm cuff of ligament still attached to divided origin of internal oblique, transversus abdominus, and transversalis fascia, may need to divide conjoint tendon at its insertion on pubis as well as anterior rectus sheath.

- Deep Dissection bluntly dissect a plane between the symphysis pubis and the bladder (space of Retzius), pack with sponges, expose anterior aspect of femoral vessels and surrounding lymphatics in midportion of incision (lacuna vasorum). Lacuna musculorum is lateral and contains iliopsoas, femoral nerve, and lateral femoral cutaneous nerve. Identify iliopectineal fascia, which seperates the lacuna vasorum and lacuna musculorum.dissect vessels and lymphatics from medial aspect of fascia, free iliopsoas and femoral nerve from lateral aspcet of fascia. sharply divide iliopectineal fascia down to pectineal eminence, then detach from pelvic brim; allows access to true pelvis, quadrilateral plate, and posterior column, place second penrose drain around iliopsoas, femoral nerve, and lateral femoral cutaneous nerve, place thrid penrose drain around femoral vessels and lymphatics, identify and ligate corona mortise before retracting vessels, subperiosteal dissection is used to expose pelvic brim, rami, and quadrilateral surface.

- Work through 3 windows to reduce and fix fracture:

- Medial window medial to external iliac artery \& vein, access to pubic rami; indirect access to internal iliac fossa and anterior SI joint.

- Middle window between external iliac vessels and the iliopsosas, access to pelvic brim, quadrilateral plate, and a portion of the superior pubic ramus.

- Lateral window lateral to iliopsoas (iliopectineal fascia), access to quadrilateral plate, SI joint, and iliac wing [5].

\section{Results}

This study was conducted among 30 patients 12 were female and 18 male with a mean age is 39.6 (Figure 1).

The diagnosis of these patients was made by using $\mathbf{x}$ rays, 3D CT scan and we found that the majority of these patients diagnosis as having anterior column fracture which represent $43 \%$ of the patients, followed by sympheseal disruption which represent $26.7 \%, 10 \%$ have left superior and inferior public rami fracture $10 \%$ also have left superior and inferior public rami fracture with anterior column fracture, followed by bilateral superior and inferior public rami sympheseal disruption, bilateral superior and inferior public rami alone, and right superior and inferior public rami which were $3.3 \%$ for each.

Among these patients we carried 3 different approach the most familial is Stoppa which represent $60 \%$ followed by modified Stoppa which represent 39.7 then illioinguinal approach that represent 3.3\%of the operation (Figure 2).

The percentage of corona mortise was found to be $13(43 \%)$ of the patient and 17 (57\%) were absent (Figure 3).

The corona mortise $\mathrm{d}$ was found to be intact in all patients that have it (Figure 4). 


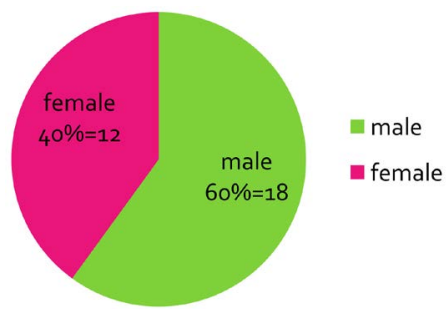

Figure 1. Sex distribution of the study group.

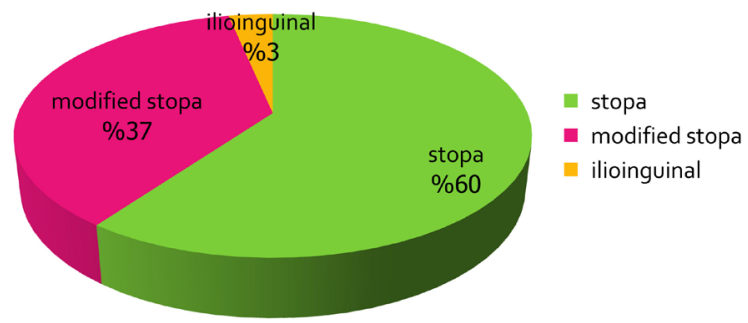

Figure 2. Approach used to treat patient with pelvic fracture of the study group.

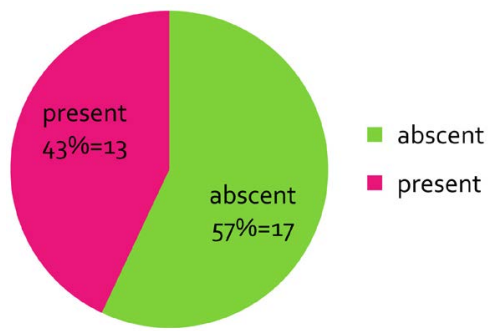

Figure 3. Presence of corona mortise in patient with pelvic fracture.

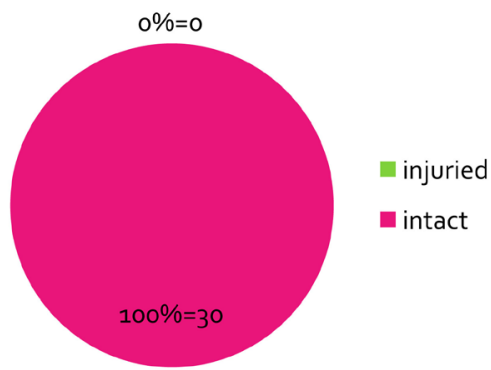

Figure 4. Status of corona mortise in the study group.

The site of corona mortise was found to be on the right side in 7 (53.8\%), 5 (38.5\%) on the left side and it is bilateral in 1 (7.7\%) (Figure 5).

The vascularity of the corona mortise was found to be arterial in 4 (30.8\%) of the cases, venous in $1(7.7 \%)$ and both arterial and venous in $8(61.5 \%)$ of the patients (Figure 6).

The size of these vessels was found to measure more than $5 \mathrm{~mm}$ in 7 (53.9\%) 3 to $5 \mathrm{~mm}$ in $4(30.8 \%)$ of the patients and less than $3 \mathrm{~mm}$ in $2(15.3 \%)$ of the patients (Figure 7).

The vessels were secured with clamp in 7 (54\%) and ligation in $6(46 \%)$ of the patients (Figure 8). 


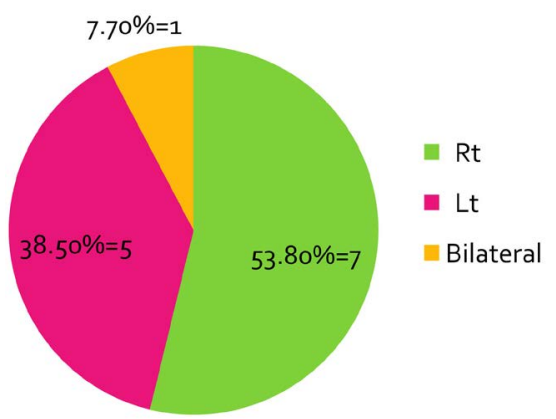

Figure 5. Site of corona mortise.

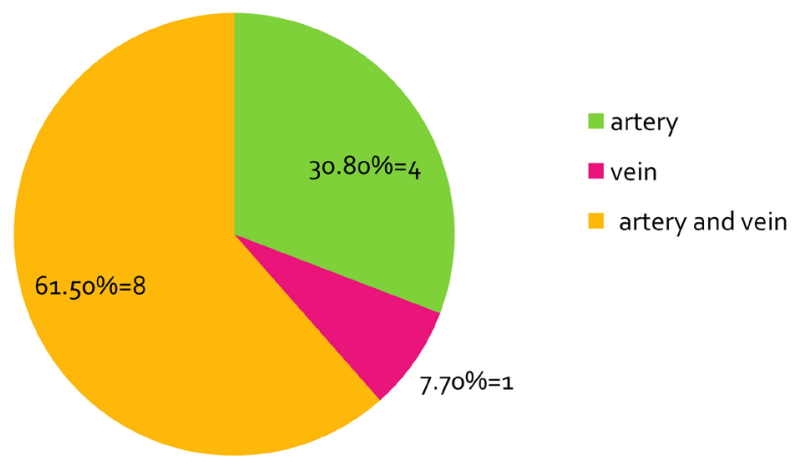

Figure 6. Vascularity of corona mortise in the study group.

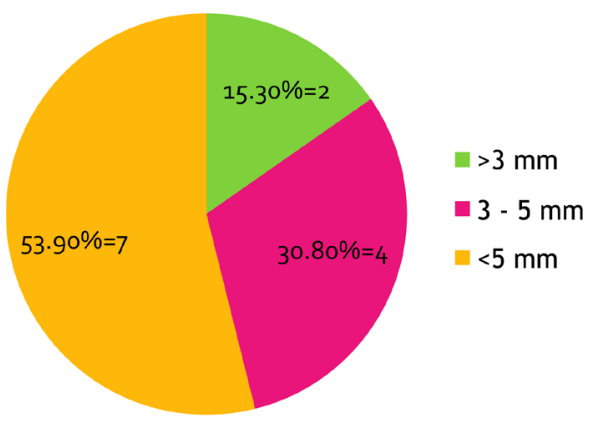

Figure 7. Size of corona mortise.

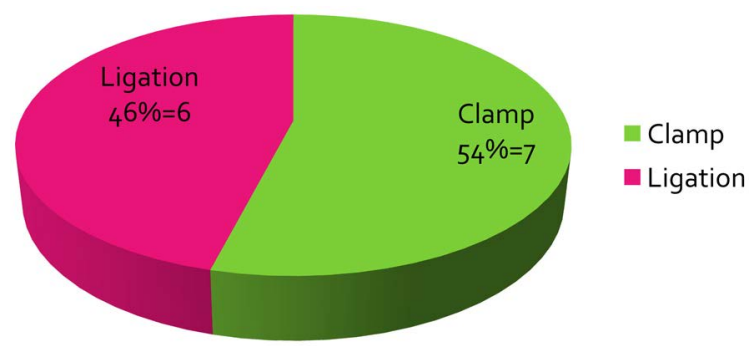

Figure 8. Secure of corona mortise during surgery.

The complication was found in $1(8 \%)$ of the patients which is accidently injury (Figure 9).

The control of the bleeding was found to be difficult in the injured patient and was clamped then ligated (Figure 10). 


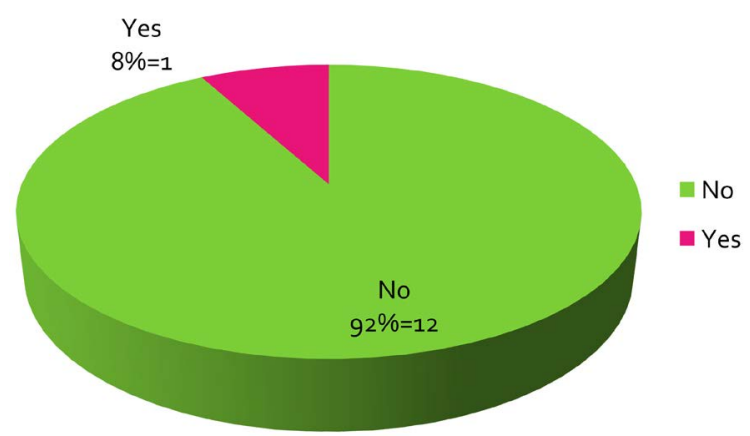

Figure 9. Presence of complications during surgery.

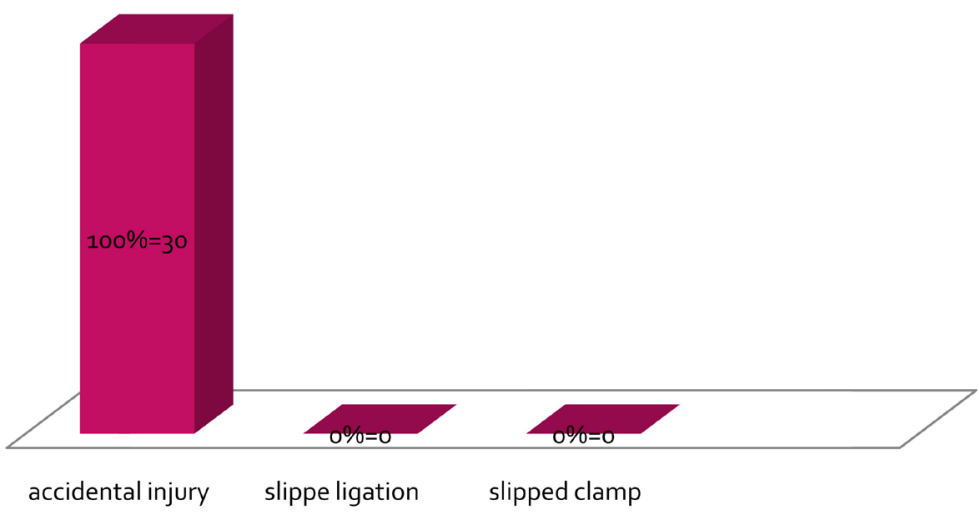

Figure 10. Complication during surgery.

\section{Discussion}

This study was conducted among 30 patients and 12 of them were female and 18 male with a mean age s $39.6 \pm 15.3$.

Among these patients we carried 3different approach the most familial is stoppa which represent $60 \%$ followed by modified stoppa which represent 39.7 then illioinguinal approach that represent $3.3 \%$ of the operation the last approach done by S. DARMANIS [1] in his study and the other authors didn't mention their approaches.

The most approach in this study was stoppa because it offers good access to acetabulam and pubic rami and it is well experienced by the surgery.

The percentage of corona mortise was fund to be 13 (43\%) of the patients which is less than what Berberoglu M, UzA, et al. [6] and S. DARMANIS [1] found in this study 33 (96\%) and 33 (83\%) and 17 (57\%) were absent in our study.

Corona mortise was found to be intact in all patients that have it.

The site of corona mortise was found to be on the right side in 7 (53.8\%), 5 (38.5\%) on the left side and it is bilateral in 1 (7.7\%) which is less than what Ely L. STEINBERG, et al. [7] found $30(45,45 \%)$ on the right side and $36(54,54 \%)$ on the left side and bilateral in $15(22,72 \%)$ and this due to the small number of samples due to the short time of study.

We can't justify if there is corona mortise or not in the other side of the pelvis 
because we just searched about it in the side of surgery.

The vascularity of the corona mortise was found to be arterial in $4(30.8 \%)$ of the cases which is in close relation S. DARMANIS [1]-DELI A, and anagnostopoulou $S$ [8] in 8 (40\%) venous in 1 (7.7\%) which is less than S. DARMANIS [1] and Stavropoulos-Deli A and anagnostopoulou S [8] 12 (50\%), and both arterial and venous in $8(61.5 \%)$ of the patients which is higher than what reported by Minnie pillay, Tintu T. sukumaran, Mahendran Mayilswamy [9] 15 (22.38\%).

The size of these was found vessels measure more than $5 \mathrm{~mm}$ in 7 (53.9\%) 3 to $5 \mathrm{~mm}$ in $4(30.8 \%)$ of the patients darmanis et al. [1] reported average artery diameter of $2.6 \mathrm{~mm}$ and $2.32 \mathrm{~mm}$ respectively we founded it to be $4.33 \mathrm{~mm}$ in diameter OKcu et al. [3] did not mention the artery diameter.

Concerning the distance from symphysis they measure it is Okcu et al. [3] 64 mm (45 - 90) darmanis et al. [1] 71 (40 - 96) and Ely L. Steinberg et al. [6] 56.2 $\mathrm{mm}(35$ - 72) we founded to be $58 \pm 16 \mathrm{~mm}$ rusu et al. [4] did not mention the distance.

The vessels were secured with clamp in 7 (54\%) and ligation in $6(46 \%)$ of patient. the compilation was found in $1(8 \%)$ of patients which is accidently injury, none of the authors mention anything about vessels benign secured and that might be because there were done in cadavers.

The control of bleeding was found to be difficult in one patient and the injured vessels was packed firstly for thirty minute then ligated and that is due delayed time of surgery adhess to surrounding soft tissue.

\section{Conclusion}

In summary, a vascular connection between the obturator and the external iliac is located over the superior public ramus. A total of 30 pelvic halves corona mortise was detected in 13 patients (43\%). the majority in the Rt hemipelvies 7 (53.8\%) speciments: arterial 4/13 (30.8\%) venous $1 / 13$ (7.7\%) and both arterial and venous in $8 / 13(61.5 \%)$ speciments respectively. Of these $53.9 \%$ had a large diameter $>5 \mathrm{~mm}$ and the largest one measure $80 \mathrm{~mm}$ with mean distance $5.8 \pm$ $1.6 \mathrm{~mm}$ thus surgery and secure the site to help in minimizing surgery time clear surgical field and prevent iatrogenic injury.

\section{Recommendation}

Surgeons who carry out an anterior approach to pelvis and acetabulum have to be cautious about corona mortise vessels at around $5.8 \pm 1.6 \mathrm{~cm}$ (range $44-80$ $\mathrm{mm}$ ) lateral to the symphysis pubis.

To do preoperative CT angiogram is helpful to knowing the site, size and type of corona mortise in both hemipelvies in the patients who have it.

To do more studies in corona mortise Sudanese population with more sample size and time.

To study the influence of delayed time of surgery from trauma and intraoperative complication. 


\section{Conflicts of Interest}

The authors declare no conflicts of interest regarding the publication of this paper.

\section{References}

[1] Darmanis, S., Lewis, A., Mansoor, A. and Bircher, M. (2007) Corona Mortise An Anatomical Study with Clinical Implication in Approach to the Pelvis and Acetabulum. Clinical Anatomy, 20, 433-439. https://doi.org/10.1002/ca.20390

[2] Hong, H.X., Pan, Z.J., Chen, X. and Huang, Z.J. (2004) An Anatomical Study of Corona Mortise and Its Clinical Significance. Chinese Journal of Traumatology, 7, 165-169.

[3] Okcu, G., Erkan, S., Yercan, H.S. and Ozic, U. (2004) The Incidence and Location of Corona Mortise: A Study on 75 Cadavers. Acta Orthopaedica Scandinavica, 75, 53 -55. https://doi.org/10.1080/00016470410001708100

[4] Rusu, M.C., Cergan, R., Motoc, A.G., folescu, R. and Pop, E. (2010) Anatomical Consideration on the Corona Mortise. Surgical and Radiologic Anatomy, 32, 17-24. https://doi.org/10.1007/s00276-009-0534-7

[5] Joshua, B. (2020) Ilioinguinal Approach to the Acetabulum. https://www.orthobullets.com/approaches/12016/ilioinguinal

[6] Berberoglu, M., Uz, A., Ozmen, M.M., Bozkurt, M.C., Erkuran, C., Taner, S. and Tekdemirl, I. (2001) Corona Mortis: An Anatomic Study in Seven Cadavers and an Endoscopic Study in 28 Patient. Surgical Endoscopy, 15, 72-75.

https://doi.org/10.1007/s004640000194

[7] Steinberg, E.L., Tomer, B.-T., Aviram, G., Steinberg, Y. and Ehud, R. (2017) Corona Mortis Anastomosis: A Three-Dimensional Computrerized Tomographic Angiographic Study. Emergency Radiology, 24, 519-523.

https://doi.org/10.1007/s10140-017-1502-x

[8] Stavropoulou-Deli, A. and Anagnostopoulou, S. (2013) Corona Mortise: Anatomical Data and Clinical Considerations. Australian and New Zealand Journal of $\mathrm{Ob}$ stetrics and Gynaecology, 53, 283-286. https://doi.org/10.1111/ajo.12076

[9] Minnie, P., Tintu, T., Sukumaran and Mahendran, M. (2017) Anatomical Consideration on Surgical Implication of Corona Mortise: An Indian Study. Italian Journal of Anatomy and Emberiology, 122, 127-137. 